\title{
Beam Positions Optimization to Achieve Improved CT Images with Limited Data
}

\author{
Rena Widita \\ Department of Physics, Faculty of Mathematics and Natural Sciences, Institut \\ Teknologi Bandung, Bandung 40132, Indonesia. E-mail: rena@fi.itb.ac.id
}

\begin{abstract}
In radiotherapy planning, margins are used to account for the uncertainties due to internal organ and patient motion as well as set-up error. Improvement in radiotherapy treatments may be achieved by reducing the set-up uncertainty and thus treatment margins allowing a higher dose to be delivered to the target volume. It is important to be able to verify the success of the treatment by determining the position of patient and the dose deposited in the patient at each fraction. One possibility for achieving this would be to collect limited information while the patient is on the treatment couch. The aim of this study is to develop a method for determining intelligent angles to use to reconstruct an image for dose verification.

A method optimizing the angles based on an objective function is required. The methods developed here are based on image correlation and projection correlation that have been investigated previously. Two optimization methods, deterministic and stochastic (simulated annealing), were also assessed. The effectiveness and practicality of each of these combinations were compared.
\end{abstract}

Keywords: image reconstruction; image correlation; projection correlation; simulated annealing.

\section{Introduction}

The determination of radiation dose distributions in the body is one of the most important steps in the radiation treatment planning process. In radiotherapy planning, dose calculation is based on computed tomography (CT). A CT for each patient is used to obtain an accurate three-dimensional model of the patient's anatomy and electron density information. To ensure accurate and consistent treatment, the patient is immobilized in the same manner as for imaging. Control images can be used to detect setup error relative to the planned position defined by a reference image. However, during the treatment, the target volume and the organs at risk may move within the patient and/or change shape. Consequently, they affect the patient setup and delivered dose from fraction to fraction. 
Margins used are often quite large to ensure sufficient dose coverage of the tumor and include organ movement. This results in higher doses delivered to the normal tissues. It is anticipated that improvements in radiotherapy treatments will be achieved by reducing the setup uncertainty and thus treatment margins, allowing a higher dose to be delivered to the target volume without affecting normal tissues. As the margins around the tumor are reduced, the ability to verify the success of the treatment by determining the dose deposited in the patient is of greater importance.

Some authors have studied image guidance in radiotherapy and have shown that a CT facility at a linear accelerator is a very valuable tool for radiotherapy setup verification ${ }^{1,2,3}$. The CT-guided procedure in fractionated radiotherapy might enable the use of tighter safety margins. To determine delivered dose for every fraction, the patient image must also be obtained for every fraction as well.

Previous research has investigated the possibility of obtaining CT images and determining delivered dose during treatment using specialized equipment ${ }^{4,5}$. The major obstacle in applying this method is the limitation of the standard radiotherapy equipment in most hospitals. In many hospitals, information from a limited number of angles can be obtained. However information from many angles, as is required with a complete CT scanner, cannot be easily obtained due to time constraints. Thus a technique for establishing a limited number of angles to be used in image reconstruction would be very useful. Furthermore, using a limited number of angles for image reconstruction, the dose to the patient can be minimized.

To obtain a patient image for every fraction, one could collect limited information while the patient is on the treatment couch. The advantage of taking a daily image while the patient is on the treatment couch is that no additional dose or very little additional dose, if a larger field size is required, will be delivered to the patient. Using standard equipment, ideally the angles from which the image projections were taken would be the same as those of the treatment. In theory, reconstruction algorithms require an infinite number of projections over the full angular range of $180^{\circ}$ for a parallel beam or $180^{\circ}+$ fan angle for a fan beam. However acceptable and useful images can be obtained with limited data. Based on this picture, the possibility of reconstructing an image with a few angles was investigated. During an image-guided procedure, the number of angles used can be far less than suggested by theoretical consideration in order to reduce scanning time and/or dose load ${ }^{6}$.

Several algorithms have been proposed in the literature to generate an image from limited projections. In image recovery, convex projection methods have been in use for almost two decades. The basic theory of convex projection was 
developed in the $1960{ }^{\prime} \mathrm{s}^{7}$. The algebraic reconstruction technique is a primitive convex projection algorithm. Using convex projection methods, a point in the intersection set can be found by iterative projection. Another author has studied the usage of megavoltage photon beams for image reconstruction ${ }^{8}$. This study explored the feasibility of reconstructing tomographic images from fan-beam projection data acquired with a commercial portal imaging device on a standard radiotherapy linear accelerator. They showed that a useful image for patient localization could be obtained from only a few projection views $(\leq 10)$ by using some of the iterative reconstruction techniques. There is still research required to improve the images achieved by solving the problem of limited view data. This could be done with a wide variety of techniques such as a Bayesian approach and maximum entropy. However, all these algorithms are highly time consuming due to the complexity of the calculation and/or iterations. Brunetti et al proposed a new algorithm based on a morphologic technique ${ }^{9}$. This method is faster that the previous methods. It can be applied with a single iteration. However, as with the other methods, the choice of angles has not been addressed. The discussed methods improve image quality for a given set of limited data often by approximating the missing data. However, they have not considered determining optimal angles to reconstruct an acceptable image. Thus, the aim of this study was to develop a method for determining the best angles to use to reconstruct an image for dose verification. It allows selection of these angles without excessive computational cost. Because of the small number of angles used, a certain level of liaising artifacts appearing in the images has to be accepted. However with the current results discussed above, although the images are of very low contrast useful information can still be extracted allowing two or more images taken at different times or from different devices to be compared.

In this work, we developed and compared two objective functions, i.e. image correlation and projection correlation, as well as methods for optimizing the angles based on those objective functions. Two optimization methods, stochastic and deterministic optimization, were also considered. The effectiveness and practicality of each of these combinations were compared.

\section{$2 \quad$ Methods and Materials}

\subsection{Projection Data Generation}

In digital medical imaging, $\mathrm{x}$-rays form an electronic image on a radiation detector. In an image matrix, the numbers in the cells represent the intensity or brightness at that location. Each cell of the image matrix is called a picture element/pixel (figure 1). The numerical value of each pixel is a CT number 
which is related to the brightness or color that can be seen when the digital image is converted into an analog image.

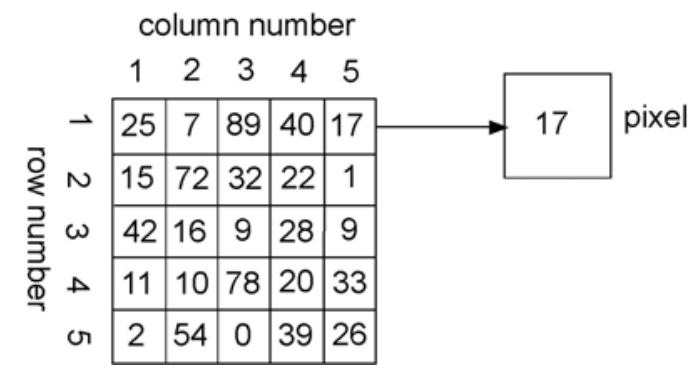

Figure 1 Digital image (a 5 by 5 matrix of numbers).

In this study, a CT image of the brain were used to generate the projection data. Each image was divided into $100 \times 100$ pixels. Each pixel was represented by a grayscale number which is between $0-255$. The projection data for a particular angle were generated by summing all pixels in the same column. The projection data generation at $0^{\circ}$ is illustrated in figure 2. These projection data were initialized as $p_{0}$. To generate projection data at $45^{0}\left(p_{45}\right)$, the matrix $a$ has to be rotated $45^{\circ}$ before summing all the pixels in the same column.

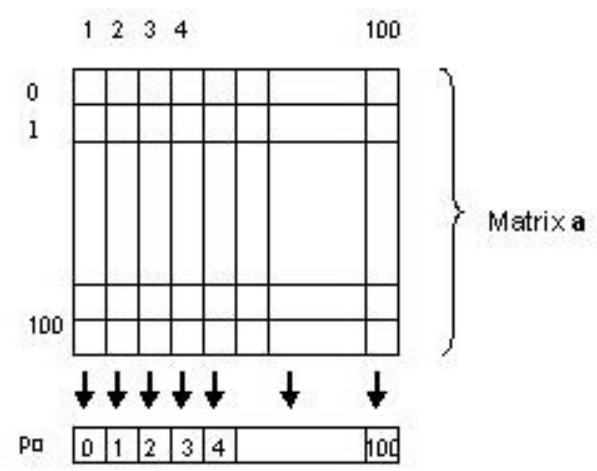

Figure 2 The concept of generating projection data for a particular angle.

\subsection{Image Reconstruction}

A CT scanner uses digitally sampled x-ray projection data from an object collected in many directions to calculate cross-sectional images of the x-ray attenuation. Reconstructing an image from a set of measurements is called image reconstruction from projections ${ }^{10}$. The image is reconstructed in the form of an array of individual pixels. 
The projection of an object at a given angle, $\theta$, is made up of a set of line integrals. The collection of all estimated line integrals is referred to as projection data. In x-ray CT, the line integrals represent the total attenuation of the beam of $x$-rays as it travels in a straight line through the object. Then, the resulting image is a 2D (or 3D) model of the attenuation coefficient. The following figure gives a simple illustration a vertical projection for a simple two-dimensional function (object) $a(x, y)$. The line integral in the horizontal direction is the projection of $a(x, y)$ onto the $y$-axis.

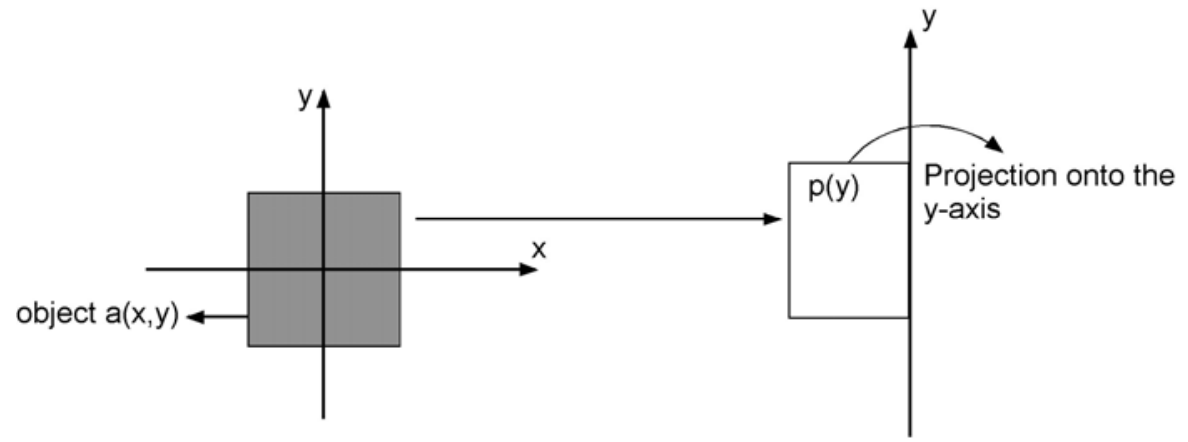

Figure 3 Projection as the line integral through an object $a(x, y)$ in the $\mathrm{x}$ direction.

If $p(y)$ is the projection through an unknown object or function $a(x, y)$ that we wish to determine, $p(y)$ can be defined as

$$
p(y)=\ln \frac{I_{0}}{I_{d}(y)}=\int \mu(x, y) d x=\int a(x, y) d x
$$

where $\mu(\mathrm{x}, \mathrm{y})$ is mass attenuation coefficient of the object. Projections can also be computed along an arbitrary angle $\theta$ (figure 4 ).

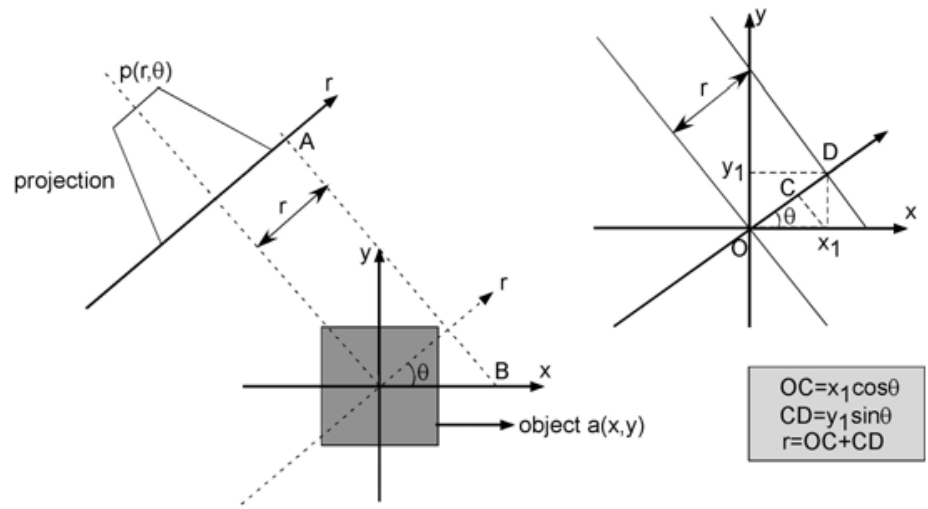

Figure 4 Parallel beam geometry at an arbitrary angle $\theta$. 
Using the coordinate system of figure 4 , the reconstruction image is obtained by adding up the images at all angles $(0-\pi)^{11}$ :

$$
a(x, y)=\int_{0}^{\pi} a^{\prime}(r, \theta) d \theta=\int_{0}^{\pi} \int_{-\infty}^{\infty} p(r, \theta) d r \delta(x \cos \theta+y \sin \theta-r) d r d \theta
$$

The process of image reconstruction can be seen in figure 5 .

\begin{tabular}{|c|c|c|c|c|}
\hline $\begin{array}{c}\mu(x, y) \text { body to } \\
\text { be imaged }\end{array}$ & $\begin{array}{c}\text { Projection } \\
\text { system }\end{array}$ & $\begin{array}{c}\mathrm{Pe}(\mathrm{t}) \\
\text { Projection data }\end{array}$ & $\begin{array}{c}\text { Image reconst } \\
\text { system }\end{array}$ & $\begin{array}{l}\text { Reconstruction } \\
\text { image }\end{array}$ \\
\hline
\end{tabular}

Figure 5 The image reconstruction processes.

The theory of reconstruction of two-dimensional functions from their projections is well-known. Several different methods or algorithms, such as transform-based, algebraic, and statistical, are in current use to solve thousands of equations to determine the linear attenuation coefficients of all the pixels in the image matrix ${ }^{10,11}$.

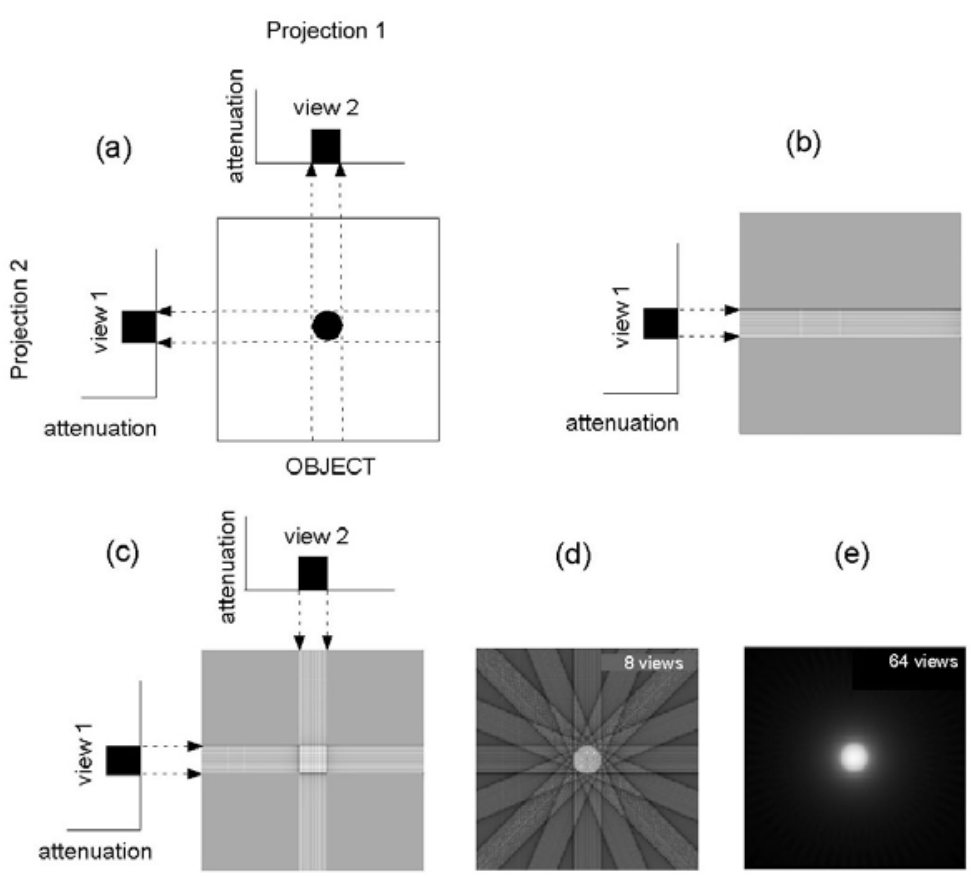

Figure 6 Principle of simple image reconstruction system. 
The simple principle of image reconstruction system (e.g. Algebraic Reconstruction Technique - ART) is described as follows. For instance we start with two scan views through a body section that contains one object. The data produced is not a complete image, but a profile of the $\mathrm{x}$-ray attenuation by the object which is known as projection data (figure 6.a). These profiles are then taken to draw an image by back-projecting the profiles onto the image surface. From the first view, there is only enough information in the profile to draw in streaks, resembling shadows across the image area (figure 6.b).

For the second view, the x-ray beam is rotated around the body by $90^{\circ}$. If this profile is now back projected onto the image area, the beginnings of an image showing the object can be seen (figure 6.c). However, two views do not give us a high-quality image. Ideally, several hundred views are used to produce clinical CT images. The back-projections are then summed, giving a reconstructed image (figure 6.e).

\subsection{Objective Function (Projection Correlation)}

The reconstructed images using a few beam positions yield a low quality image. An objective function to rank the image quality is required to automate the decision process in determining the optimum beam positions. It was developed that projection correlation could be used as the objective function ${ }^{12}$. The goal of this objective function (projection correlation) was to process data at a reasonable computational cost while still achieving acceptable results. The algorithm used here is geometry-free algorithms and thus do not require particular source-detector geometry such as in parallel-beam or fan beam CT. Projection correlation is a method of estimating the degree to which a group of projections are correlated. In projection correlation, it is expected that the correlation would be low for good images. This is because a set of projections which contain dissimilar information (uncorrelated) to each other would give a superior result compared with those containing similar information to each other (correlated) for the final image. Thus by minimizing the projection correlation, the reconstructed image will converge toward the sought-after original image. The concept of the projection correlation has been described in details in another journal ${ }^{13}$.

Based on the work of previous authors, it was found that about 7 or 9 beams give satisfying results in all cases for treatment planning optimization ${ }^{14,15}$. However, these numbers of angles are unlikely to be the optimal solution for image reconstruction, although they have produced improved images in this work. Determining the ideal number of angles for image reconstruction when trying to reduce the overall number of angles was not considered in this work. 


\section{$2.4 \quad$ Optimization}

To establish a limited number of beam positions with the goal of improved image reconstruction in an automated and time efficient fashion, two optimization algorithms employing the projection correlation as the objective functions were proposed. The search for the best possible beam configuration within a given search domain will include different numbers of configurations from 1 to infinity. Due to the massive number of possible configurations and the possibility that the global minimum is hidden in many local minima, a stochastic optimization is proposed here as the first optimization method. One of the best stochastic algorithms to find the global minimum is simulated annealing. This method is suitable for problems with large scale, in this case the number of configurations. Simulated annealing is not a method that is time efficient. However, implementing many tricks, such as choosing a good annealing schedule and/or objective function, can make this process much faster. As a comparison, a deterministic optimization was also proposed, which is also based on the projection correlation for the second optimization method. This will not necessarily determine the optimal values, however it was anticipated that it would determine acceptable values. The deterministic optimization was then compared to the stochastic optimization in terms of computational cost and resulting images.

\subsubsection{Beam Positions Determination Based on Simulated Annealing}

The concept of simulated annealing was independently invented by Kirkpatrick in $1983^{16}$. Simulated annealing is a generalization of a Monte Carlo method for examining the equations of state and frozen states of n-body systems ${ }^{17}$. The concept is based on the manner in which liquids freeze or metals recrystalize in the process of annealing. In an annealing process a melt, initially at high temperature and disordered, is slowly cooled so that the system at any time is approximately in thermodynamic equilibrium. As cooling proceeds, the system becomes more ordered and approaches a "frozen" ground state at $\mathrm{T}=0$. If the initial temperature of the system is too low or cooling is done insufficiently slowly the system may become quenched forming defects or freezing out in metastable states (ie. trapped in a local minimum energy state).

Simulated annealing optimization starts with a Metropolis Monte Carlo simulation at a high temperature. This means that a relatively large percentage of the random steps that result in an increase in the energy will be accepted. After a sufficient number of Monte Carlo steps, or attempts, the temperature is decreased. This process is repeated until the final temperature is reached.

A sequence of solutions are generated by randomly creating a new solution through a perturbation to the current solution and then accepting or rejecting the 
new point with a certain probability which is dependent on the temperature and the change in the objective function. The probability of accepting a proposed move, known as the acceptance probability (Metropolis criterion), is given by the expression

$$
P_{\text {accept }}(\Delta f, T)= \begin{cases}1 & \text {;if } \Delta f \leq 0 \\ e^{-(\Delta f) / T} & \text {;if } \Delta f>0\end{cases}
$$

Here $\Delta \mathrm{f}$ is the change in the cost function corresponding to the proposed change in state, and $T$ is the artificial control parameter representing temperature. The fact that the acceptance probability is exactly 1 when $\Delta \mathrm{f}$ is negative indicates that moves which improve the cost are always accepted. However, simulated annealing may accept cost increases, enabling the algorithm to escape from local minima.

Initial Value. The concept of temperature is completely arbitrary. As mentioned above, a very high initial temperature results in a lot of time being wasted at the beginning stage of the search. However, a very low initial temperature could result in the system being trapped in a local potential energy minimum, because the configuration space was not sufficiently explored. The initial value of the control parameter $\mathrm{T}_{0}$ should be large enough to allow virtually all transitions to be accepted, so that about $80 \%$ of all possible transitions are accepted. This temperature is strongly dependent on the number of beam positions, measured data, and the initial image.

Decrement Rules. The temperature should decreases sufficiently slowly so the system will not be trapped in local minimum. The value of control parameter used to decrease the temperature is based on the decrement function which is given by ${ }^{18}$ :

$$
T_{k+1}=\alpha T_{k} \quad ; k=1,2, \ldots
$$

where $\alpha=$ a constant smaller but close to 1 (typical values lie between 0.8 and 0.99). The flowchart of the optimization of beam positions based on simulated annealing can be seen in figure 7 .

The simulated annealing algorithm is very time consuming, but it promises to succeed in that it can find the global optimum more accurately than other techniques. Although it is possible to find the global minimum, the parameters in this algorithm must be carefully set to ensure this is achieved. The algorithm terminates when some specified stopping criterion is met, e.g. when no improvement has been found for some number of moves. When this stopping criterion is fulfilled the algorithm gives as a result the current value. 


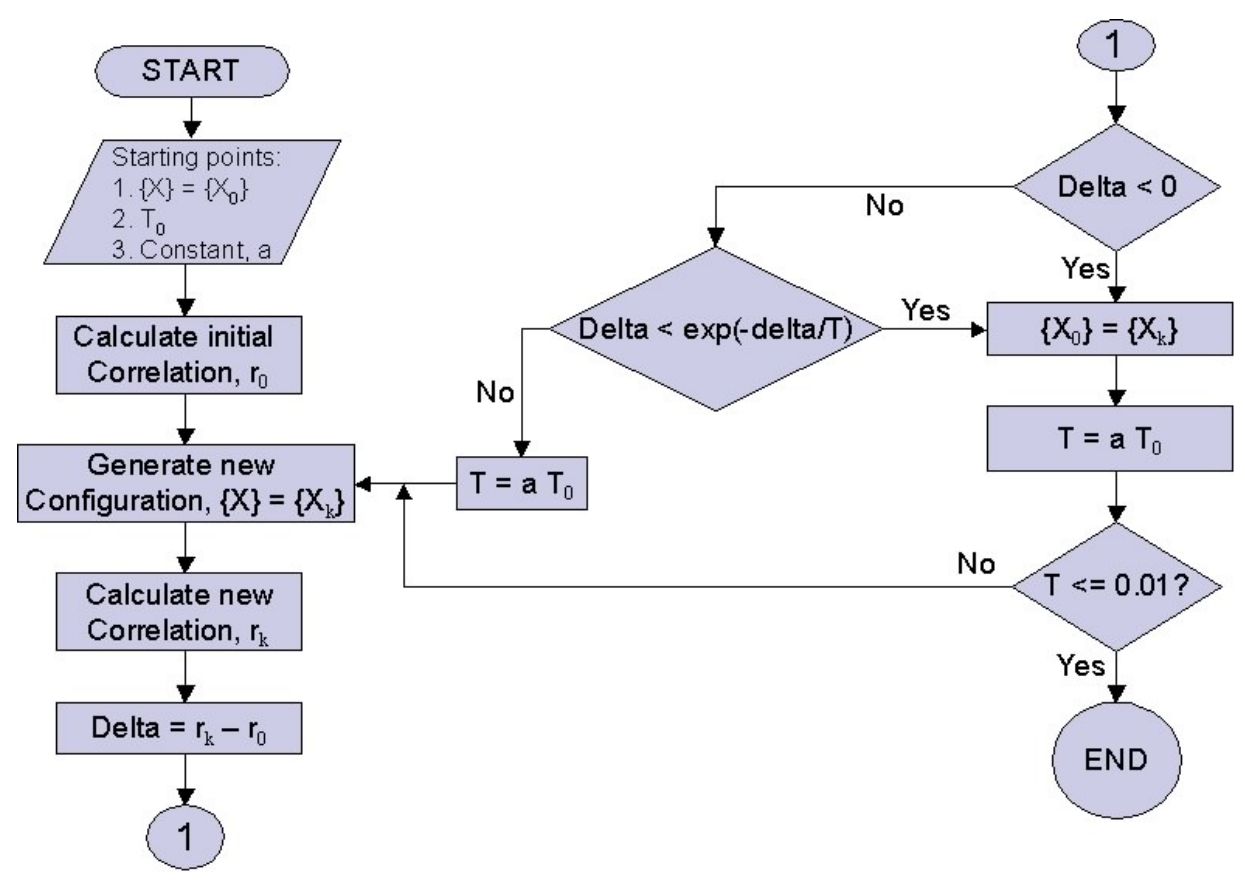

Figure 7 The flowchart of simulated annealing in relation to the projection correlation.

\subsubsection{Beam Positions Determination Based on Deterministic Optimization}

The approach of this algorithm is to find the beam configuration based on projection correlation from the first angle chosen. Consider 360 projection data equally spaced at $1^{\circ}$ angular increments. To yield a solution with $\mathrm{n}$ beams, we first choose the first beam and then the remaining beams are chosen by minimizing the projection correlation between or among the group of beams. Let the first beam be $B_{1}$, the second beam is then searched for within $0^{\circ}-360^{\circ}$ $\left(\mathrm{B}_{2} \neq \mathrm{B}_{1}\right)$, determining the angle which will give the smallest correlation value (minimize $\mathrm{P}_{12}$ ). $\mathrm{P}_{12}$ is defined as

$$
P_{12}=r_{12}
$$

where $r_{12}$ is the correlation between the first and the second projection. The third angle can be found by applying the same method. $\mathrm{B}_{3}$ is determined by searching within $0^{\circ}-360^{\circ}\left(\mathrm{B}_{3} \neq \mathrm{B}_{1} \neq \mathrm{B}_{2}\right)$ to find the smallest value for correlation $\mathrm{P}_{13}$.

$$
P_{13}=r_{12}+r_{13}+r_{23}=P_{12}+r_{13}+r_{23}
$$


Then, the solution with $\mathrm{n}$ beams can be found by minimizing the projection correlation value one by one up to $n$ beams $\left(P_{n}\right)$. The concept of this deterministic algorithm can be seen in figure 8 .

To establish the optimal angles, both optimization methods, stochastic and deterministic, were applied using the projection correlation as the objective function. The effectiveness and practicality of each of these combinations were compared. The number of beam positions used is 7 and 9 which is chosen from 12,15 and 360 angles (the angles were selected equally at $30^{\circ}, 24^{\circ}$, and $1^{\circ}$ increments respectively).

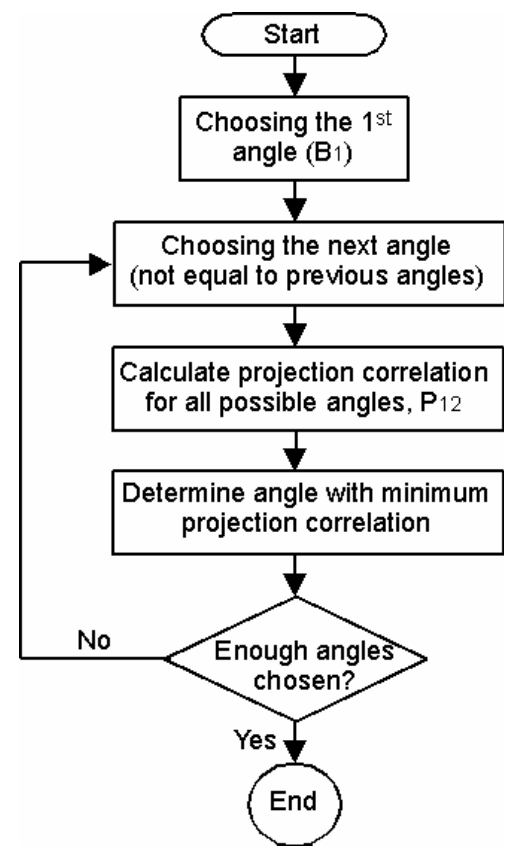

Figure 8 Flowchart of angle determination based on deterministic optimization.

\section{$3 \quad$ Results and Discussion}

It can be seen in figure 9 and 10 that based on the projection correlation value (PC), the simulated annealing optimization is superior to the deterministic optimization. The advantage of the deterministic algorithm is it is much faster (about 9 times) than the simulated annealing. However the results show that there is a probability that the solution is trapped in local minima when using the deterministic optimization. The time required for simulated annealing depends on the number of iterations used (the initial temperature and the final temperature. The time required for this work was determined using 200 degree 
as the initial temperature and 0.01 degree for the final temperature. The beam positions for 7 and 9 can be seen in table 1 and 2 respectively.

Using the projection correlation as an objective function and the deterministic algorithm as the optimizer, the solution for determining angles can be much faster. The fast process of the projection correlation ensures this method could be applied for all clinical cases. This algorithm could potentially be used in a clinical environment together with more advanced image reconstruction.

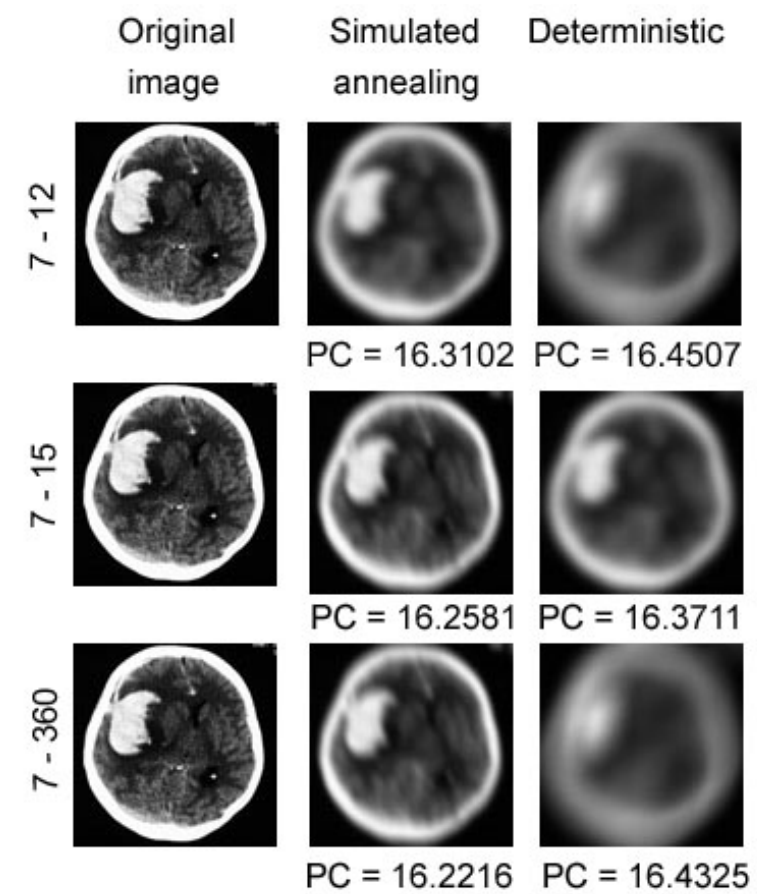

Figure 9 Simulated annealing versus deterministic optimization for 7 beams using the $\mathrm{PC}$ as the objective function.

Tabel 1 The optimum beam position for 7 beams.

\begin{tabular}{|c|c|l|l|}
\hline \multirow{2}{*}{ MODEL } & \multirow{2}{*}{ COMBINATION } & \multicolumn{1}{|c|}{ BEAM POSITION } \\
\cline { 3 - 4 } & & \multicolumn{1}{|c|}{ Simulated Annealing } & \multicolumn{1}{c|}{ Deterministic } \\
\hline \multirow{5}{*}{ Brain } & $7-12$ & $0,90,150,180,210$, & $0,30,90,180,270,300$, \\
\cline { 2 - 4 } & $7-15$ & 270,300 & 330 \\
\cline { 2 - 4 } & & $0,96,192,216,264$, & $0,24,96,192,264,312$, \\
& $7-360$ & 312,336 & $0,10,129,203,255$, \\
\cline { 2 - 4 } & & 268,336 & $0,33,90,145,201,270$, \\
\hline
\end{tabular}




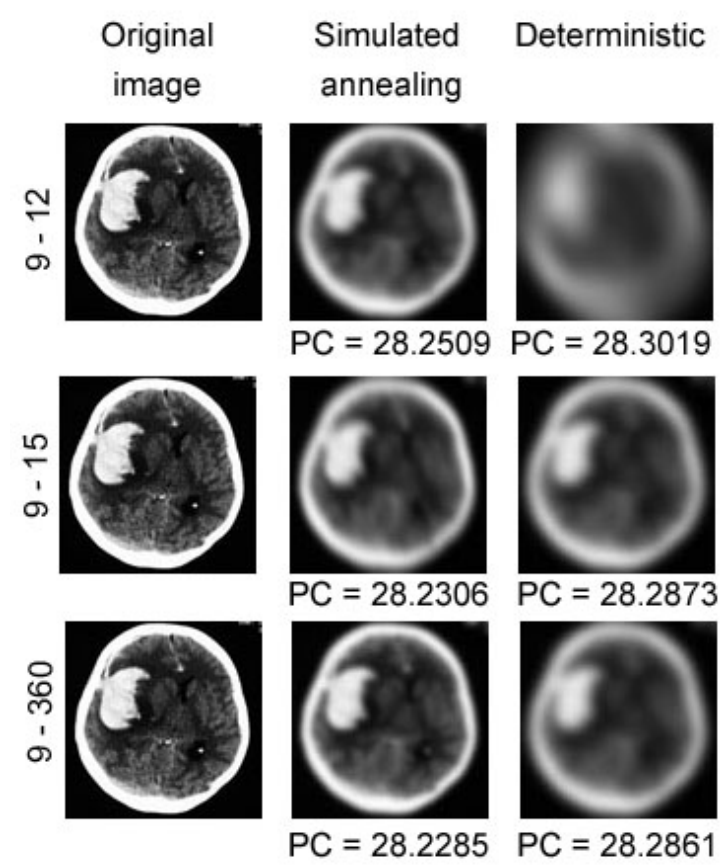

Figure 10 Simulated annealing versus deterministic optimization for 9 beams using the $\mathrm{PC}$ as the objective function.

Tabel 2 The optimum beam position for 9 beams.

\begin{tabular}{|c|c|c|c|}
\hline \multirow{2}{*}{ MODEL } & \multirow{2}{*}{ COMBINATION } & \multicolumn{2}{|c|}{ BEAM POSITION } \\
\cline { 2 - 4 } & & Simulated Annealing & Deterministic \\
\hline \multirow{4}{*}{ Brain } & $9-12$ & $0,30,90,120,150,180$, & $0,30,90,150,180$, \\
& & $210,270,330$ & $210,270,300,330$ \\
\cline { 2 - 4 } & $9-15$ & $0,24,96,168,192,216$, & $0,24,96,168,192$, \\
& & $264,312,336$ & $216,264,312,336$ \\
\cline { 2 - 4 } & $9-360$ & $0,10,28,49,93,115$, & $0,33,90,145,180$, \\
& & $266,327,344$ & $201,270,307,340$ \\
\hline
\end{tabular}

\section{Conclusion}

The simulated annealing optimization produced superior results to the deterministic optimization, even though the simulated annealing algorithm is slower than the deterministic optimization. The combination of the simulated annealing and projection correlation algorithm is very promising. It can reduce the searching time and results in a better solution. 
A detailed comparison of these calculation results with previous analytic studies indicates that the projection correlation may enable the determination of optimum angles for individual treatment plans. It works most effectively when fewer angles are selected from a particular number of angles, thus more variations are possible. For example, the results of 7-360 are superior to those of 100-360. Together with more advanced image reconstruction software these algorithms could potentially be used in a clinical environment where selecting relatively few angles for a large initial number will be the likely scenario.

\section{References}

1. Hesse, B. M., Spies, L. \& Groh, B. A., Tomotherapeutic Portal Imaging for Radiation Treatment Verification, Physics in Medicine and Biology, 43, 3607-3616 (1998).

2. Jaffray, D. A., Drake, D. G., Moreau, M., Martinez, A. A. \& Wong, J. W., A Radiographic and Tomographic Imaging System Integrated Into A Medical Linear Accelerator for Localization Of Bone And Soft-Tissue Targets, International Journal of Radiation Oncology Biology Physics, 45, 773-789 (1999).

3. Ruchala, K. J., Olivera, G. H., Schloesser, E. A. \& Mackie, T. R., Megavoltage CT on a tomotherapy system. Physics in Medicine and Biology, 44, 2597-2621 (1999).

4. Kapatoes, J. M., Olivera, G. H., Balog, J. P., Keller, H., Reckwerdt, P. J. \& Mackie, T. R., On the accuracy and effectiveness of dose reconstruction for tomotherapy, Physics in Medicine and Biology, 46, 943-966 (2001).

5. Kapatoes, J. M., Olivera, G. H., Reckwerdt, P. J., Fitchard, E. E., Schloesser, E. A. \& Mackie, T. R., Delivery verification in sequential and helical tomotherapy, Physics in Medicine and Biology, 44, 1815-1841 (1999).

6. Wiesent, K., Barth, K., Navab, N., Durlak, P., Brunner, T., Schuetz, O. \& Seissler, W., Enhanced 3-D-reconstruction algorithm for C-arm systems suitable for interventional procedures, Medical Imaging, IEEE Transactions on, 19, 391-403 (2000).

7. $\quad$ Oskoui-Fard, P. \& Stark, H., Tomographic Image Reconstruction Using the Theory of Convex Projections, Medical Imaging, IEEE Transactions on, 7, 45-58 (1988).

8. Loose, S. \& Leszcynski, K. W., On few-view tomographic reconstuction with megavoltage photon beams, Medical Physics, 28, 1679-1688 (2001).

9. Brunetti, A. \& Golosio, B., A new algorithm for computer tomographic reconstruction from partial view projections, Medical Physics, 28, 462468 (2001). 
10. Herman, G. T., Image Reconstruction from Projection: The Fundamental of Computerized Projections, Academic Press (1980).

11. Kak, A. C. \& Slaney, M., Principles of computerized tomographic imaging, IEEE Press (1999).

12. Widita, R. \& Holloway, L. C., A method for determining angles to achieve improved CT images with limited data, EPSM 2004 (Engineering and Physical Scientists in Medicine Australasian Conference), Geelong, Victoria, Australia (2004).

13. Widita, R., A method for beam positions determination in image reconstruction, Indonesian Journal of Physics (submitted) (2006).

14. Bortfeld, T., Bürkelbach, J., Boesecke, R. \& Schlegel, W., Methods of image reconstruction from projections applied to conformation radiotherapy, Physics in Medicine and Biology, 35, 1423-1434 (1990).

15. Brahme, A., Optimization of stationary and moving beam radiation therapy techniques, Radiotherapy and Oncology, 12, 129-140 (1988).

16. Kirkpatrick, S., C.D. Gelatt, J. \& Vecchi, M. P., Optimization by Simulated Annealing, Science, 220, 671-680 (1983).

17. Metropolis, N., Rosenbluth, A., Rosenbluth, M., Teller, A. \& Teller, E., Equation of State Calculations by Fast Computing Machines, Journal of Chemical Physics, 21, 1087-1092 (1953). 